\title{
Perioperative management in distal pancreatectomy: results of a survey in 23 European participating centres of the DISPACT trial and a review of literature
}

\author{
Helge Bruns ${ }^{\dagger 1}$, Nuh N Rahbari ${ }^{\dagger 1}$, Thorsten Löffler ${ }^{1}$, Markus K Diener ${ }^{1,2}$, \\ Christoph M Seiler ${ }^{1,2}$, Matthias Glanemann³, Giovanni Butturini ${ }^{4}$, \\ Christoph Schuhmacher ${ }^{5}$, Inga Rossion², Markus W Büchler*1, \\ Tido Junghans ${ }^{6}$ and the DISPACT Trial group
}

Address: ${ }^{1}$ Department of General, Visceral and Transplantation Surgery, Ruprecht-Karls-University Heidelberg, Im Neuenheimer Feld 110, 69120 Heidelberg, Germany, ${ }^{2}$ Study Centre of the German Surgical Society (SDGC), Ruprecht-Karls-University Heidelberg, Im Neuenheimer Feld 110, 69120 Heidelberg, Germany, ${ }^{3}$ Department of General, Visceral, and Transplantation Surgery, Universitätsmedizin Berlin, Augustenburger Platz 1, Charité Campus Virchow Klinikum, 13353 Berlin, Germany, ${ }^{4}$ Policlinico Borgo Roma, Universita di Verona, Piazzale La Scuro 10, 37134 Verona, Italy, ${ }^{5}$ Department of General Surgery, Technische Universität München, Ismaningerstrasse 22, Munich 81675, Germany and ${ }^{6}$ Department of General, Visceral, Vascular and Thoracic Surgery, Universitätsmedizin Berlin, Charitéplatz 1, Charité Campus Mitte, 10117 Berlin, Germany

Email: Helge Bruns - helge.bruns@med.uni-heidelberg.de; Nuh N Rahbari - nuh.rahbari@med.uni-heidelberg.de;

Thorsten Löffler - thorsten.loeffler@med.uni-heidelberg.de; Markus K Diener - markus.diener@med.uni-heidelberg.de; Christoph M Seiler - christoph.seiler@med.uni-heidelberg.de; Matthias Glanemann - matthias.glanemann@charite.de; Giovanni Butturini - giovanni.butturini@univr.it; Christoph Schuhmacher - schuhmacher@chir.med.tu-muenchen.de; Inga Rossion - inga.rossion@med.uni-heidelberg.de; Markus W Büchler* - markus.buechler@med.uni-heidelberg.de;

Tido Junghans - Tido.Junghans@klinikum-bremerhaven.de; the DISPACT Trial group - markus.buechler@med.uni-heidelberg.de

* Corresponding author †Equal contributors

Published: 26 July 2009

Trials 2009, 10:58 doi:10.1186/1745-6215-10-58

This article is available from: http://www.trialsjournal.com/content//0/I/58

(C) 2009 Bruns et al; licensee BioMed Central Ltd.

This is an Open Access article distributed under the terms of the Creative Commons Attribution License (http://creativecommons.org/licenses/by/2.0), which permits unrestricted use, distribution, and reproduction in any medium, provided the original work is properly cited.
Received: 13 November 2008

Accepted: 26 July 2009

\begin{abstract}
Background: Concomitant treatment in addition to intervention may influence the primary outcome, especially in complex interventions such as surgical trials. Evidence-based standards for perioperative care after distal pancreatectomy, however, have been rarely defined. This study's objective was therefore to identify and analyse the current basis of evidence for perioperative management in distal pancreatectomy.
\end{abstract}

Methods: A standardised questionnaire was sent to 23 European centres recruiting patients for a randomized controlled trial (RCT) on open distal pancreatectomy that would compare suture versus stapler closure of the pancreatic remnant (DISPACT trial, ISRCTN I8452029). Perioperative strategies (e.g., bowel preparation, pain management, administration of antibiotics, abdominal incision, drainages, nasogastric tubes, somatostatin, mobilisation and feeding regimens) were assessed. Moreover, a systematic literature search in the Medline database was performed and retrieved meta-analyses and RCTs were reviewed.

Results: All 23 centres returned the questionnaire. Consensus for thoracic epidural catheters (TECs), pain treatment and transverse incisions was found, as well as strong consensus for the placement of intra-abdominal drainages and perioperative single-shot antibiotics. Also, there was consensus that bowel preparation, somatostatin application, postoperative nasogastric tubes and intravenous feeding might not be beneficial. The literature search identified 16 meta-analyses and 
19 RCTs demonstrating that bowel preparation, somatostatin therapy and nasogastric tubes can be omitted. Early mobilisation, feeding and TECs seem to be beneficial for patients. The value of drainages remains unclear.

Conclusion: Most perioperative standards within the centres participating in the DISPACT trial are in accordance with current available evidence. The need for drainages requires further investigation.

Trial registration: Clinical trial registration: ISRCTN 18452029

\section{Background}

Short-term outcomes after major abdominal surgery are influenced by indication, surgical intervention, the surgeons' expertise, hospital volume and perioperative management [1-5]. In order to qualitatively assess the impact of the various factors on outcome, a rigorous means of evaluation such as the randomized controlled trial (RCT) design is necessary. Mortality and morbidity in pancreatic surgery has decreased in high-volume centres in recent years $[6,7]$ mainly by virtue of standardised surgical and optimised perioperative management [8]. In high volume centres, mortality from distal pancreatectomy is as low as $5 \%$ while morbidity remains as high as $40 \%$ [7,9-12]. Morbidity is determined by perioperative factors such as bowel preparation, incision type, analgesia, mobilisation, and feeding regimens, amongst other factors [1]. In addition, long-term survival after pancreatectomy as cancer treatment strongly depends on a centre's operative volume [13].

A recent study of 617 consecutive pancreatic resections demonstrated local complications to be more frequent than systemic complications [14]. Thus, the formation of pancreatic fistulas can be considered the most important complication after a pancreatic left resection. Currently, the Study Centre of the German Surgical Society is conducting a large RCT (DISPACT trial, ISRCTN 18452029) to compare the occurrence rates of pancreatic fistula after stapler versus hand-sewn closure of the pancreas after left resections [15]. Both expert opinions and evidence-based standards were taken into account in the development of the study protocol. The consensus-assisted development of study protocols has proven to be beneficial and may indeed be helpful in identifying when consensus is present but not justified by evidence [16]. All 23 centres

Table I: Total pancreatectomies and distal pancreatic resections performed in 2008 at centres taking part in DISPACT Trial

\begin{tabular}{|c|c|c|c|}
\hline Centre & Number of pancreatic resections & Number of distal pancreatectomies & $\begin{array}{r}\text { Number of patients randomized in } \\
2008\end{array}$ \\
\hline Amsterdam, Netherlands & 76 & 12 & 8 \\
\hline Berlin Charité Mitte, Germany & 20 & 12 & 4 \\
\hline Berlin Charité Virchow, Germany & 197 & 48 & 20 \\
\hline Berlin Lichtenberg, Germany & 38 & 10 & 4 \\
\hline Bochum St. Josef, Germany & 214 & 46 & 7 \\
\hline Dresden-Friedrichstadt, Germany & 52 & 5 & 2 \\
\hline Freiburg, Germany & 83 & 15 & 2 \\
\hline Gent, Belgium & 68 & 6 & 3 \\
\hline Heidelberg, Germany & 423 & 87 & 45 \\
\hline Homburg, Germany & 61 & 10 & - \\
\hline Cologne-Merheim, Germany & 25 & 10 & - \\
\hline Liverpool, Great Britain & 128 & 13 & I \\
\hline Ljubljana, Slovenia & 90 & 25 & 10 \\
\hline Mannheim, Germany & 71 & 10 & 4 \\
\hline Marburg, Germany & 48 & 13 & I \\
\hline Munich-LMU, Germany & 91 & 19 & 13 \\
\hline Munich-TU, Germany & 87 & 27 & 22 \\
\hline Regensburg, Germany & 70 & 19 & 6 \\
\hline Verona, Italy & 269 & 66 & 17 \\
\hline Würzburg, Germany & 20 & 7 & 2 \\
\hline Wuppertal, Germany & 24 & 3 & I \\
\hline Total & 2155 & 463 & 172 \\
\hline Median (range) & $71(20-423)$ & $13(3-87)$ & $4(0-45)$ \\
\hline
\end{tabular}


contributing to DISPACT are centres with a substantial caseload of pancreatic left resection (Table 1). The study protocol of DISPACT has been approved by the ethics committees of all participating centres and has been recently published [15]. Since the DISPACT trial aims to evaluate the effect of two surgical procedures (stapler and hand-sewn closure) on the development of pancreatic fistula, surgical and perioperative standardisation is of special interest. The present study was launched during an investigator meeting to identify the current practices concerning perioperative management amongst the various participating centres. This study aims to identify the level of consensus of relevant factors in perioperative management. In addition, an extensive literature search was conducted to compare findings with current best available evidence.

\section{Methods}

\section{Survey of current practice}

Twenty-three European centres participating in the DISPACT trial were evaluated using a standardized questionnaire to evaluate perioperative management [see Additional file 1]. Standards in bowel preparation, pain and feeding management, antibiotics prophylaxis, incision type, drainages, necessity and duration of intensive care, mobilisation and perioperative somatostatin therapy were assessed. These relevant items were identified in a previously published meta-analysis and included in the questionnaire [17]. The survey was designed by T.L., N.N.R. and C.M.S and mailed to all centres taking part in the DISPACT trial. The survey was completed and returned by all local surgical sub-investigators. Consensus levels were classified for each outcome as published elsewhere [18]. Briefly, a discrete scale was used and the results were stratified along consensus levels $1-4$, which in turn corresponded to being standard in $<50 \%$ (no consensus), $50-75 \%$ (overall agreement), $75-95 \%$ (consensus), and $>95 \%$ (strong consensus) of participating centres, respectively.

For time-critical factors such as the removal of intraabdominal drainages, thoracic epidural catheters (TECs) and gastric tubes; the discharge from intensive care unit (ICU) or intermediate care (IMC) wards; and the duration of intravenous feeding and somatostatin therapy, median time and interquartile ranges (IQRs) have been calculated.

\section{Literature search of best practice}

Only RCTs or meta-analyses of RCTs assessing perioperative care were eligible for inclusion. Since specific evidence of perioperative care for distal pancreatectomy and pancreatic surgery was expected to be low, articles on general abdominal surgery including hepatic and colorectal surgery were included as well and evaluated in hierarchical order (distal pancreatectomy > pancreatic surgery > abdominal surgery > hepatic and colorectal surgery). Studies of paediatric, laparoscopic, and transplantation surgery were excluded. A search algorithm was developed and an extensive systematic Medline search using Boolean operator functions and wildcards (i.e. the asterisk symbol) was performed independently by three authors of this study (H.B., N.N.R. and T.L.). The search algorithm used single relevant keywords, medical subject headings (MESH), and their combinations (Appendix 1). No other literature databases than Medline were searched and a subsequent analysis of the identified literature was performed. All published articles were evaluated.

Reference lists of the retrieved literature were manually cross-searched for additional publications independently by three researchers (T.L., N.N.R and H.B.) under the supervision of an experienced researcher (C.M.S.). Moreover, captured citations were filtered for study design in order to identify all RCTs and meta-analyses. Titles, abstracts, and full text articles were screened for the selection of relevant studies.

\section{Results}

\section{Survey of current practice}

All 23 centres participating in the DISPACT trial returned completely filled out questionnaires either per fax, mail or email. The annual caseload of pancreatic resections for each centre within the year 2008 is given in Table 1. All centres performed at least 20 pancreatic resections, which was the requirement for participation in the DISPACT trial. Pancreatic left resections (median 13; range 3-87) are performed less commonly than other resections. Only two centres have not randomized patients for DISPACT within the last year. Median 4 patients (range 0-45) were randomized. The random ratio (randomized left resections/total left resection * 100) was $37 \%$.

\section{Bowel preparation}

Mechanical bowel preparation (MBP) as a standard was performed at eight of the 23 participating centres (Table 2 ). Enemas were made use of at seven centres and one centre performed orthograde lavage; 14 hospitals did not use any kind of MBP before left pancreatectomy (Table 2).

\section{Type of incision, intraoperative antibiotics and placement of abdominal drainages}

The transverse incision was exclusively used by 16 hospitals and the midline by four hospitals. Two centres used other types of incisions and one centre used both incisions, depending on the individual surgeon's choice (Table 2). Intraoperative single-shot antibiotics were performed in all but one centre (Table 2).

All centres placed intra-abdominal drainages. Drains were left for median of four days (IQR 3-5), with a maximum of eight days at one centre (Table 2). 
Table 2: Perioperative standards in the 23 European centres for pancreatic surgery

\begin{tabular}{|c|c|c|c|c|}
\hline & $\mathrm{n}$ & $\%$ & consensus level & median duration (IQR) \\
\hline \multicolumn{5}{|l|}{ Bowel preparation } \\
\hline none & 15 & 65 & overall agreement & - \\
\hline enema & 7 & 30 & & - \\
\hline orthograde lavage & I & 4 & & - \\
\hline \multicolumn{5}{|l|}{ Type of incision } \\
\hline Midline ${ }^{+}$ & 5 & 22 & & - \\
\hline Transverse $^{+}$ & 18 & 78 & consensus & - \\
\hline Other & 2 & 4 & & - \\
\hline \multicolumn{5}{|c|}{ Intraoperative single-shot antibiotic prophylaxis } \\
\hline No & 1 & 4 & & - \\
\hline Yes & 22 & 96 & strong consensus & - \\
\hline \multicolumn{5}{|c|}{ Intra-abdominal drainages } \\
\hline No & 0 & 0 & & \\
\hline Yes & 23 & 100 & strong consensus & $4(3-5)$ \\
\hline \multicolumn{5}{|l|}{ Postoperative care } \\
\hline IMC*** & 9 & 39 & & $2(I-2.25)$ \\
\hline ICU*** & 11 & 48 & & $1(1-1)$ \\
\hline Nursery ward & 5 & 22 & & - \\
\hline Not specified & I & 4 & & - \\
\hline \multicolumn{5}{|c|}{ Pain management (thoracic epidural catheter) } \\
\hline No & 3 & 13 & & - \\
\hline Yes* & 20 & 87 & consensus & $4(3-5)$ \\
\hline \multicolumn{5}{|c|}{ Post-operative gastric tube } \\
\hline No & 13 & 57 & overall agreement & - \\
\hline Yes & 10 & 43 & & $\mid(\mid-2)$ \\
\hline \multicolumn{5}{|l|}{ Intravenous feeding } \\
\hline No & 12 & 52 & overall agreement & - \\
\hline Yes & 11 & 48 & & $2(1.5-2)$ \\
\hline \multicolumn{5}{|l|}{ Somatostatin therapy } \\
\hline No & 15 & 65 & overall agreement & - \\
\hline Yes & 8 & 35 & & $6(3.75-7)$ \\
\hline
\end{tabular}

+ One centre reported using both midline and transversal incisions frequently.

*Local anaesthetics and opioids were used at $75 \%$ of the centres $(n=15), 15 \%(n=3)$ used local anaesthetics without opioids, and $10 \%(n=2)$ did not specify administered drugs.

** Patients were transferred from the ICU to the IMC at three centres. While there is no consensus whether patients should be transferred to the ICU or IMC, there is an overall agreement in $74 \%$ of centres $(n=17)$ that patients should not be returned to nursery wards immediately after distal pancreatectomy.

\section{Postoperative care}

Postoperatively, 17 centres transferred their patients to ICU or IMC wards, whereas five centres transferred patients directly to nursery wards. Patients were discharged from ICU and IMC after a median of one day (IQR 1-1) and two days (IQR 1-2.25), respectively (Table 2).

\section{Pain management}

Thoracic epidural catheters (TECs) were used in 20 hospitals and kept in place for a median duration of four days (IQR 3-5) (Appendix 1). Fifteen centres used a combination of local anaesthetics and opioids for the medication of TECs and three centres used exclusively local anaesthetics (Appendix 1). Two centres did not specify the medication.

\section{Nasogastric tube and feeding regimens}

Postoperatively, a nasogastric tube was placed for a median of one day (IQR 1-2) in 10 hospitals (Table 2). Eleven centres used parenteral feeding for a median of two days (IQR 1.5-2) (Table 2). Oral feeding with fluids started on day 1 (IQR (0-1) and solid food on day 2 (IQR 2-3) (Table 3). One centre reported keeping patients on parenteral feeding till day 5 and starting oral feeding with fluids and solids on day 5 .

\section{Mobilisation}

Mobilisation of patients started at postoperative day 1 (IQR 0-1) (Table 3). Eight centres started mobilisation on the operative day, while 14 centres started on first postoperative day. One centre reported starting mobilisation on the second postoperative day. 
Table 3: Mobilisation and oral feeding

\begin{tabular}{lc}
\hline Begin of... & postoperative day (median (IQR)) \\
\hline ...mobilisation & $\mathrm{I}(0-1)$ \\
...oral feeding & Fluids \\
Solid food & $\mathrm{I}(0-1)$ \\
\hline
\end{tabular}

\section{Somatostatin therapy}

Fifteen centres did not use Somatostatin as a routine after pancreatic surgery. In eight centres, somatostatin was used as a standard after left pancreatectomy for a median of six days (IQR 3.75-7) (Appendix 1).

\section{Literature search of best practice}

All of the identified studies focus on large bowel surgery. Besides pancreatic surgery, other procedures have been included based on the similarity in concepts of perioperative management and determinants of complications.

A total of 16 meta-analyses of RCTs and 19 RCTs were identified and reviewed [see Additional file 2].

\section{Bowel preparation}

We identified two meta-analyses $[19,20]$ which included 16 RCTs and six RCTs [21-26] with a total number of 3046 patients and 3747 patients, respectively [see Additional file 2]. All of these studies failed to identify a benefit from bowel preparation; both meta-analyses $[19,20]$ and two of the six RCTs $[21,25]$ found beneficial effects of no bowel preparation in terms of decreased anastomotic leakage and/or wound infections. Slim et al. [19] and Guenaga et al. [20] identified a rate of $6.3 \%$ vs. $3.2 \%$ (p = $0.003)$ and $5.6 \%$ vs. $3.2 \%(p=0.032)$ for bowel preparation versus no bowel preparation, respectively [see Additional file 2].

\section{Type of incision}

While complication rates were not different in the metaanalysis by Brown et al. [27] in transversal versus midline incisions, a recent RCT by Fassiadis et al. [28] identified a decreased risk of incisional hernia after transverse incision for abdominal aortic aneurysm repair only $(\mathrm{p}=0.01$; [see Additional file 2]. Moreover, Brown et al. [27] suggested that transverse incisions may be less painful (odds ratio [OR] for less analgesic use (mg morphine equivalent) for total hospital stay, $-6.29 ; 95 \%$ CI [-12.57; -0.01]) and may affect pulmonary function less than midline access (OR for percentage change in forced expiratory volume in one second on the last day of measurement, 18.31 ; 95\% CI $[6.84 ; 29.78])$.

\section{Perioperative antibiotic prophylaxis}

Regarding antibiotic prophylaxis, the meta-analysis by Song et al. [29], which included 147 RCTs, showed single- shot antibiotics to be as effective as long-term antibiotics in the prevention of surgical wound infections [see Additional file 2]. The recent study by Fujita et al. [30] identified a dose-dependent correlation between surgical site infections and antibiotics ( $\mathrm{p}=0.009$; [see Additional file 2].

\section{Abdominal drainages}

Gurusamy et al. [31] included five trials and 465 patients in their meta-analysis [see Additional file 2]. No significant influence of drainages on complication rates after liver resection was found [see Additional file 2] [31]. Petrowsky et al. [32] were able to identify surgical procedures after which abdominal drainages significantly decrease complications (i.e. oesophageal resection and total gastrectomy) and in which abdominal drainages can be omitted (i.e. hepatic, rectal or colonic resection with primary anastomosis, and appendectomy) [see Additional file 2]. In a RCT by Conlon et al. [33], no correlation between the placement of abdominal drainages and the need for interventional drainages and surgical exploration after septic complications in patients undergoing pancreatectomy was detected [see Additional file 2]. For hepatectomy, Sun et al. [34] were able to identify a correlation of abdominal drainages and postoperative wound complications in a RCT ( $28 \%$ vs. $3 \%, \mathrm{p}<0.001$; [see Additional file 2].

\section{Pain management}

In their meta-analysis of 16 RCTs, Marret et al. [35] did not identify any differences in length of hospitalisation between patients receiving epidural or parenteral analgesia after colorectal surgery [see Additional file 2]. Werawatganon et al. [36], who included 711 patients from nine RCTs in their analysis, and Wu et al. [37] identified better pain relief with epidural analgesia compared to intravenous analgesia [see Additional file 2]. In a metaanalysis by Jørgensen et al. [38], a decreased rate of gastrointestinal paralysis with comparable effect on pain was found for epidural local anaesthetics compared to opioidbased medication [see Additional file 2]. In RCTs, Flisberg et al. [39] and Mann et al. [40] were able to identify better pain relief with epidural analgesia and less frequent side effects compared to i.v. analgesia during rest ( $p<0.003)$ and mobilisation $(\mathrm{p}<0.001)$ [39] and rest $(\mathrm{p}<0.001)$ and coughing $(\mathrm{p}<0.002)[40]$, respectively [see Additional file 2].

\section{Nasogastric tubes}

Two meta-analyses by Nelson et al. [41,42] did not identify any advantage of routinely placed gastric tubes after abdominal surgery [see Additional file 2]. Additionally, an earlier return of bowel function was found in patients without gastric tube in both studies $(\mathrm{p}<0.001)[41,42]$ [see Additional file 2]. Pesseaux et al. [43] included 200 patients after hepatic resection in a RCT and did not find 
an advantage for nasogastric tubes. They demonstrated an increased risk of pulmonary complications (i.e. pneumonia and atelectasis, $\mathrm{p}=0.047$ and $\mathrm{p}=0.043$, respectively) within the nasogastric tube group [see Additional file 2] [43].

\section{Feeding regimens}

Andersen et al. [44] included 13 RCTs with 1173 patients in their meta-analysis. No advantage for late return to oral diet was found [see Additional file 2] [44]. Han-Geurts et al. [45], who included 128 patients in a RCT on early (i.e. within the first two postoperative days) versus late (i.e. more than two days after procedure) postoperative return to oral diet, also failed to identify a significant difference in or beneficial effect of keeping patients starved in terms of postoperative ileus and recovery [see Additional file 2].

\section{Mobilisation}

Three RCTs [46-48] on postoperative mobilisation and physiotherapy were available [see Additional file 2]. While Browning et al. [46] concluded that early mobilisation may decrease hospitalisation length, Mackay et al. [48] did not find a benefit of chest physiotherapy in high risk patients after open abdominal surgery as far as pulmonary complications are concerned [see Additional file 2]. In the larger study by Fagevik Olsén et al. [47], chest physiotherapy decreased postoperative pulmonary complications and improved the mobilisation of patients $(6 \%$ in physiotherapy group vs. $29 \%$ in control group; $\mathrm{p}<$ 0.001 ; [see Additional file 2].

\section{Somatostatin therapy}

While Connor et al. [49] and Alghamdi et al. [50] identified a reduced complication rate in their meta-analyses on somatostatin versus no somatostatin after pancreatic surgery $(p=0.003$ [49] and $p=0.004$ [50]), Zeng et al. [51] did not find any significant effect on morbidity [see Additional file 2]. All included meta-analyses [49-51] failed to identify any effect of somatostatin on mortality rates [see Additional file 2].

No beneficial effect of somatostatin was found in all included RCTs $[52,53]$ on somatostatin after pancreatic surgery [see Additional file 2]. Hesse et al. [52] did not identify any reduced rate of pancreatic fistula. Even worse, Shan et al. [53] in their study found delayed gastric emptying to be more frequent in the somatostatin group.

\section{Discussion}

Surgical and perioperative management remains a matter of debate. Besides surgical expertise and technique $[9,13]$, perioperative procedures significantly influence morbidity $[1,6]$. This survey of 23 centres participating in a large multicenter trial on pancreatic left resection revealed that general perioperative standards can be detected in pancre- atic surgery. In most items, at least overall agreement was present. Strong consensus was found in two items (i.e. placement of abdominal drainages and intraoperative antibiotic prophylaxis) and consensus was present for the usage of TECs for pain medication and transversal incisions as the abdominal cavity opening (Table 2). The DISPACT centres agreed overall that no bowel preparation, gastric tube, somatostatin therapy or intravenous feeding was necessary after left pancreatectomy (Table 2).

While most of the strategies and standards in the DISPACT group are in accordance with current evidence, this survey has detected some differences in the perioperative management after pancreatic left resection despite current available evidence from meta-analyses and RCTs.

\section{Bowel preparation}

As early as 1972, Hughes [54] has questioned the beneficial effect of bowel preparation in large-bowel surgery. However, this tradition has remained a standard in abdominal surgery for decades. Within the DISPACT group, there was overall agreement that no bowel preparation is necessary before pancreatic left resection (Appendix 1). Based on the results from our literature research, this standard is in accordance with current evidence. Recent meta-analyses [19,20] and RCTs [21-26] did not identify any benefit from bowel preparation before abdominal surgery (Table 3A). In both meta-analyses by Guenaga et al. and by Slim et al. [19,20], an increased rate of anastomotic leakage was identified. Moreover, bowel preparation may even be disadvantageous in terms of wound infections [19-21].

In eight (35\%) of 23 DISPACT centres, bowel preparation was used as a standard prior to left pancreatectomy. This is in contrast to the results of studies conducted in colorectal surgery demonstrating no significant differences for anastomotic leakage rates and overall morbidity [see Additional file 2] [19-26]. While there is no evidence that enema or orthograde lavage may be harmful in pancreatic surgery, it seems likely that there is no benefit from bowel preparation. Therefore, bowel preparation can no longer be considered as a necessary standard in pancreatic surgery. Most DISPACT centres have therefore already stopped any bowel preparation.

\section{Type of incision}

A recent meta-analysis did not show any significant difference between midline and transverse incision as far as complication rates, incisional hernias and recovery times [see Additional file 2] [27,28]. Amongst the DISPACT centres, there was consensus $(78 \%)$ that a transverse incision may be more beneficial for distal pancreatectomy (Table 2). While the meta-analysis by Brown et al. [27] failed to identify any difference between groups with midline and 
transverse abdominal incisions, Fassiadis et al. [28] found increased rates of incisional hernia in patients undergoing surgery for aortic aneurysms after midline versus transversal incision ( 20 of 22 patients versus 6 of 15 patients, respectively). This finding is confounded by the underlying connective tissue disorders which is a causative agent for both diseases and therefore may not be relevant for benign and malignant pancreatic disorders [55-57]. Therefore, the practise of the DISPACT centres is in accordance with the evidence.

\section{Antibiotic prophylaxis}

Antibiotic prophylaxis significantly reduces surgical site infections as long as local tissue concentrations reach an effective level [see Additional file 2] [29,30]. Both singleshot and long-term antibiotics are equally effective. As a result, antibiotic prophylaxis is a common procedure in surgery.

All but one centre agreed on using single-shot antibiotics before left pancreatectomy (Appendix 1). Given the ubiquitous use of antibiotic prophylaxis in surgery, it can be suspected that one centre chose a different regime and may have used a long-term antibiotic prophylaxis.

\section{Abdominal drainages}

Placement of drainages has a long tradition in abdominal surgery and can be dated back to the $19^{\text {th }}$ century. While many surgical procedures originating from these times have been omitted, Lawson Taits advice of "when in doubt, drain" is still considered standard in many surgical centres [58]. Not surprisingly, all centres taking part in the DISPACT trial agreed on using intra-abdominal drainages as a standard after distal pancreatectomy (Table 2). There were considerable differences in time concerning the removal of drainages, though; the median day of removal was the fourth postoperative day (IQR 3-5). Some centres kept drainages as a standard for up to eight days.

The detection of postoperative pancreatic fistulas according to the consensus definition requires a drainage for at least three days [59]. Since the occurrence of pancreatic fistula is the primary endpoint of the DISPACT trial, placement of abdominal drainages had to be included as a standard in the study protocol. Unfortunately, there is evidence that intra-abdominal drainages are not beneficial in all cases and sometimes may even be harmful [see Additional file 2] [31-34]. For a number of procedures (i.e. liver resection, appendectomy and colonic or rectal resection with primary anastomosis), there is no need for abdominal drainages while for other procedures (i.e. oesophageal resection or total gastrectomy) there are proven benefits [32]. Moreover, wound complications may even be more common when drainages are placed [34].
A recent RCT by Conlon et al. [33] failed to show a decreased mortality or rate of complications by intraperitoneal drainages after pancreatectomy [see Additional file 2]. A total number of 179 patients were included. Forty of them underwent distal pancreatectomy; data was consistent among the different procedures in pancreatic surgery. No reduced need for interventional drainages and surgical exploration for septic complications was found in the drainage group [33].

In summary, the value of drainages in pancreatic surgery remains unclear. Further studies are necessary to resolve the currents uncertainties regarding drainages after pancreatic surgery.

\section{Postoperative care}

Most centres (74\%) agree that patients undergoing distal pancreatectomy should be transferred to ICU or IMC wards (Table 2). In 22\% of the DISPACT centres, patients are transferred directly to regular nursery wards. No metaanalyses or RCTs were identified in our Medline research regarding this topic.

\section{Pain management}

Pain management is generally considered a key factor of fast track concepts in surgery and a determinant of hospitalisation length $[60,61]$. In $20(87 \%)$ of the DISPACT centres, a thoracic epidural catheter was used for pain management. Fifteen centres (75\%) applied regimens consisting of local anaesthetics and opioids (Table 2). While overall time of hospitalisation is not significantly different between patients receiving epidural and parenteral opioids, epidural administration significantly decreases side effects [see Additional file 2] [35-37,39,40]. In RCTs published by Filsberg et al. [39] and Mann et al. [40], epidural versus intravenous application of pain medication was compared [see Additional file 2]. Opioidrelated gastrointestinal side effects especially are less frequent in epidural analgesia groups $[39,40]$. In the study by Jørgensen et al. [38], no difference in pain relief could be detected between opioid-based analgesia and analgesia using epidural application of local anaesthetics only. Within the DISPACT group, thoracic epidural catheters (TEC) were clearly preferred for pain management and placed for median of four days (IQR 3-3, Table 2). The preference for opioids in this context seems to be questionable.

\section{Nasogastric tubes}

Most DISPACT centres do not apply post-operative gastric tubes as a standard after distal pancreatectomy (Table 2). Ten centres (43\%) keep nasogastric tubes in place for a median of one day (IQR 1-2) (Table 2). Both meta-analyses and RCTs with a total number of 9634 included patients could not detect benefits from nasogastric tubes 
after abdominal surgery [see Additional file 2] [41-43] Contrary to traditional belief, gastric tubes as a postoperative standard seem to be associated with a higher risk of pulmonary complications and delayed return of bowel function [see Additional file 2] [41-43]. Postoperative removal of nasogastric tubes is a standard based on evidence.

\section{Feeding regimens}

Administration of oral fluids was performed on median postoperative day 1 (IQR $0-1$ ) and of solid food on day 2 (IQR 2-3) (Table 3). Most centres did not perform intravenous feeding (Table 2). Both the RCT by Han-Geurts et al. [45] and the meta-analyses by Andersen et al. [44] did not find any benefit in keeping patients starved after abdominal surgery [see Additional file 2]. Since an early (i.e. second postoperative day) return to an oral diet is not associated with increased postoperative complications, there is no rationale in keeping patients starved [see Additional file 2] [44,45].

\section{Mobilisation}

Early postoperative patient mobilisation (median postoperative day 1 (IQR $0-1$ ) is performed in most of the centres (Table 3 )). While early mobilisation actually may reduce length of hospitalisation, the value of postoperative chest physiotherapy remains unclear [see Additional file 2] [46-48]. Mackay et al. [48] could not detect any significant decrease in pulmonary complications with chest physiotherapy in high risk patients. Contrary to this, Fagevik et al. [47] reported a significant decrease in postoperative pulmonary complications and an improved mobilisation with chest physiotherapy [see Additional file 2].

\section{Somatostatin therapy}

Fifteen (65\%) DISPACT centres do not use somatostatin as a postoperative standard (Appendix 1). All reviewed studies agree that prophylactic somatostatin does not decrease mortality [see Additional file 2] [49-53,62]. As far as meta-analyses are concerned, the effect of somatostatin on the occurrence of pancreatic fistula remains unclear [49-51]. All reviewed RCTs not only agree that somatostatin does not have any influence on occurrence of pancreatic fistula, but Shan et al. [53] also found increased rates of delayed gastric emptying after prophylactic somatostatin administration and provided decreased plasma motilin levels as a possible mechanism [see Additional file 2] [52,62]. Therefore, somatostatin as a postoperative standard should be avoided.

\section{Conclusion}

Evidence-based perioperative management is an important cornerstone for a successful outcome after complex surgical procedures. The results of this survey and the comparison with the current available evidence detected some inconsistencies in perioperative management of patients with pancreatic left resection. At least overall agreement was present in most items and was - with the exception of abdominal drainages - in accordance with strategies that were identified as beneficial for the patients from our literature research. Evidence-based perioperative treatment in pancreatic surgery requires the further conduct of randomized controlled trials.

\section{Abbreviations}

DISPACT: DIStal PAnCreaTectomy; ICU: Intensive Care Unit; IMC: InterMediate Care; IQR: Inter Quartile Range; ISRCTN: International Standard Randomized Controlled Trial Number; MBP: Mechanical Bowel Preparation; MESH: Medical Subject Headings; RCT: Randomized Controlled Trial; TEC: Thoracic Epidural Catheter.

\section{Competing interests}

The authors declare that they have no competing interests.

\section{Authors' contributions}

HB performed the analysis, literature research and drafted the manuscript. NRR and TL performed the survey and literature search. MKD and TJ designed the study. CMS designed the study and reviewed the manuscript. MG, GB, CS, IR and MWB reviewed and contributed substantially to the manuscript. All authors read and approved the final manuscript.

\section{Appendix I \\ Items and corresponding search algorithms used for Medline search of literature-based standards Bowel preparation}

(Pancreas surgery $[\mathrm{tw}]$ OR pancreas resection $[\mathrm{tw}] \mathrm{OR}$ pancreatic surgery [tw] OR pancreatic resection [tw] OR distal pancreatectomy [tw] OR pancreatic left resection [tw] OR pancreaticoduodenectomy [tw] OR hepatic surgery $[\mathrm{tw}]$ OR hepatic resection $[\mathrm{tw}]$ OR liver surgery $[\mathrm{tw}]$ OR hepatectomy $[\mathrm{tw}]$ OR colorectal surgery $[\mathrm{tw}]$ OR colon resection [tw] OR colon surgery [tw] OR abdominal surgery [tw] OR gastrointestinal surgery [tw]) AND (Mechanical bowel preparation [tw] OR bowel preparation [tw] OR bowel cleansing [tw] OR colon preparation [tw]) AND (randomized controlled trial [pt] OR controlled clinical trial [pt] OR randomized controlled trials [MESH] OR random allocation [MESH] OR double-blind method [MESH] OR single-blind method [MESH] OR clinical trial [pt] OR clinical trials [MESH] OR ("clinical trial" [tw]) OR ((singl* ${ }^{*}$ tw $]$ OR doubl* ${ }^{*}$ tw] OR tripl* $\left.{ }^{*}[\mathrm{tw}]\right)$ AND (mask* [tw] OR blind* $[\mathrm{tw}]))$ OR placebos [MESH] OR placebo* [tw] OR random* [tw] OR research design [mh:noexp] OR Meta-Analysis [ptyp] OR systematic review [tw] NOT (animal [MESH] NOT human [MESH]) NOT case reports [pt] NOT Comment [PT] NOT letter [PT] NOT child 
[MESH] NOT infant [MESH] NOT child [MESH] NOT infant $[\mathrm{MESH}])$

\section{Thoracic epidural catheter}

((Pancreas surgery [tw] OR pancreas resection [tw] OR pancreatic surgery $[\mathrm{tw}]$ OR pancreatic resection [tw] OR distal pancreatectomy [tw] OR pancreatic left resection [tw] OR pancreaticoduodenectomy [tw] OR hepatic surgery $[\mathrm{tw}]$ OR hepatic resection [tw] OR liver surgery [tw] OR hepatectomy [tw] OR colorectal surgery [tw] OR colon resection [tw] OR colon surgery [tw] OR abdominal surgery $[\mathrm{tw}]$ OR gastrointestinal surgery [tw]) AND (Thoracic epidural catheter $[\mathrm{tw}]$ OR epidural catheter* $[\mathrm{tw}]$ OR epidural analgesia [MESH] OR epidural opioid [tw] OR epidural local anaesthetic [tw]) AND (randomized controlled trial [pt] OR controlled clinical trial [pt] OR randomized controlled trials [MESH] OR random allocation [MESH] OR double-blind method [MESH] OR single-blind method [MESH] OR clinical trial [pt] OR clinical trials [MESH] OR ("clinical trial" [tw]) OR ((singl* [tw] OR doubl* [tw] OR tripl* ${ }^{*}$ tw] $)$ AND (mask* [tw] OR blind* [tw])) OR placebos [MESH] OR placebo* [tw] OR random* [tw] OR research design [mh:noexp] OR MetaAnalysis [ptyp] OR systematic review [tw] NOT (animal [MESH] NOT human [MESH]) NOT case reports [pt] NOT Comment [PT] NOT letter [PT] NOT child [MESH] NOT infant [MESH])

\section{Somatostatin}

(Gastrointestinal surgery [tw] OR pancreas surgery [tw] OR pancreas resection [tw] OR pancreatic surgery [tw] OR pancreatic resection [tw] OR distal pancreatectomy [tw] OR pancreatic left resection [tw] OR pancreaticoduodenectomy [tw]) AND (somatostatin [tw] OR octreotide [tw] OR vapreotide [tw] OR lanreotide [tw]) AND (randomized controlled trial [pt] OR controlled clinical trial [pt] OR randomized controlled trials [MESH] OR random allocation [MESH] OR double-blind method [MESH] OR single-blind method [MESH] OR clinical trial [pt] OR clinical trials [MESH] OR ("clinical trial" [tw]) OR ((singl* [tw] OR doubl* [tw] OR tripl* [tw]) AND (mask* [tw] OR blind* [tw])) OR placebos [MESH] OR placebo* [tw] OR random* [tw] OR research design [mh:noexp] OR Meta-Analysis [ptyp] OR systematic review [tw] NOT (animal [MESH] NOT human [MESH]) NOT case reports [pt] NOT Comment [PT] NOT letter [PT] NOT child [MESH] NOT infant [MESH])

\section{Incision}

(Surgery [MESH]) AND (midline incision [tw] OR transverse incision [tw] OR abdominal incision [tw] OR midline laparotomy [tw] OR transverse laparotomy [tw]) AND (randomized controlled trial [pt] OR controlled clinical trial [pt] OR randomized controlled trials [MESH] OR random allocation [MESH] OR double-blind method
[MESH] OR single-blind method [MESH] OR clinical trial [pt] OR clinical trials [MESH] OR ("clinical trial" [tw]) OR ((singl* ${ }^{*}[\mathrm{tw}]$ OR doubl* ${ }^{*}$ tw $]$ OR tripl* ${ }^{*}$ tw $]$ ) AND (mask* [tw] OR blind* [tw])) OR placebos [MESH] OR placebo* [tw] OR random* [tw] OR research design [mh:noexp] OR Meta-Analysis [ptyp] OR systematic review [tw] NOT (animal [MESH] NOT human [MESH]) NOT case reports [pt] NOT Comment [PT] NOT letter [PT] NOT child [MESH] NOT infant [MESH])

\section{Antibiotic prophylaxis}

(Pancreas surgery [tw] OR pancreas resection [tw] OR pancreatic surgery [tw] OR pancreatic resection [tw] OR distal pancreatectomy [tw] OR pancreatic left resection [tw] OR pancreaticoduodenectomy [tw] OR hepatic surgery $[\mathrm{tw}]$ OR hepatic resection [tw] OR liver surgery [tw] OR hepatectomy $[\mathrm{tw}]$ OR colorectal surgery [tw] OR colon resection [tw] OR colon surgery [tw] OR abdominal surgery [tw] OR gastrointestinal surgery [tw]) AND (Antibiotic prophylaxis [tw] OR single shot [tw] OR antimicrobial prophylaxis [tw]) AND (randomized controlled trial [pt] OR controlled clinical trial [pt] OR randomized controlled trials [MESH] OR random allocation [MESH] OR double-blind method [MESH] OR singleblind method [MESH] OR clinical trial [pt] OR clinical trials [MESH] OR ("clinical trial" [tw]) OR ((singl* [tw] OR doubl* [tw] OR tripl* [tw]) AND (mask* [tw] OR blind* [tw])) OR placebos [MESH] OR placebo* [tw] OR random* [tw] OR research design [mh:noexp] OR MetaAnalysis [ptyp] OR systematic review [tw] NOT (animal [MESH] NOT human [MESH]) NOT case reports [pt] NOT Comment [PT] NOT letter [PT] NOT child [MESH] NOT infant [MESH] NOT child [MESH] NOT infant [MESH])

\section{Nasogastric tube}

(Pancreas surgery [tw] OR pancreas resection [tw] OR pancreatic surgery $[\mathrm{tw}]$ OR pancreatic resection [tw] OR distal pancreatectomy [tw] OR pancreatic left resection [tw] OR pancreaticoduodenectomy [tw] OR hepatic surgery [tw] OR hepatic resection [tw] OR liver surgery [tw] OR hepatectomy $[\mathrm{tw}]$ OR colorectal surgery $[\mathrm{tw}]$ OR colon resection [tw] OR colon surgery [tw] OR abdominal surgery $[\mathrm{tw}]$ OR gastrointestinal surgery [tw]) AND (Nasogastric decompression [tw] OR nasogastric tube [tw]) AND (randomized controlled trial [pt] OR controlled clinical trial [pt] OR randomized controlled trials [MESH] OR random allocation [MESH] OR double-blind method [MESH] OR single-blind method [MESH] OR clinical trial [pt] OR clinical trials [MESH] OR ("clinical trial" [tw]) OR ((singl* $\left.{ }^{*} \mathrm{tw}\right]$ OR doubl* $\left.{ }^{[\mathrm{tw}}\right]$ OR tripl* $\left.{ }^{*}[\mathrm{tw}]\right)$ AND (mask* [tw] OR blind* $[\mathrm{tw}])$ ) OR placebos [MESH] OR placebo* [tw] OR random* [tw] OR research design [mh:noexp] OR Meta-Analysis [ptyp] OR systematic review [tw] NOT (animal [MESH] NOT human [MESH]) NOT case reports [pt] NOT Comment [PT] NOT letter [PT] NOT child 
[MESH] NOT infant [MESH] NOT child [MESH] NOT infant [MESH])

\section{Abdominal drainage}

(Surgery [MESH]) AND (abdominal drain* [tw] OR intraperitoneal drain* [tw] OR intraabdominal drain* [tw] OR prophylactic drain* [tw]) AND (randomized controlled trial [pt] OR controlled clinical trial [pt] OR randomized controlled trials [MESH] OR random allocation [MESH] OR double-blind method [MESH] OR single-blind method [MESH] OR clinical trial [pt] OR clinical trials [MESH] OR ("clinical trial" [tw]) OR ((singl* [tw] OR doubl $^{*}[\mathrm{tw}]$ OR tripl* $\left.{ }^{*} \mathrm{tw}\right]$ ) AND (mask* [tw] OR blind* [tw])) OR placebos [MESH] OR placebo* [tw] OR random* [tw] OR research design [mh:noexp] OR MetaAnalysis [ptyp] OR systematic review [tw] NOT (animal [MESH] NOT human [MESH]) NOT case reports [pt] NOT Comment [PT] NOT letter [PT] NOT child [MESH] NOT infant [MESH] NOT child [MESH] NOT infant [MESH])

\section{Postoperative feeding}

(Pancreas surgery [tw] OR pancreas resection [tw] OR pancreatic surgery $[\mathrm{tw}]$ OR pancreatic resection [tw] OR distal pancreatectomy [tw] OR pancreatic left resection [tw] OR pancreaticoduodenectomy [tw] OR hepatic surgery $[\mathrm{tw}]$ OR hepatic resection [tw] OR liver surgery [tw] OR hepatectomy [tw] OR colorectal surgery [tw] OR colon resection [tw] OR colon surgery [tw] OR abdominal surgery [tw] OR gastrointestinal surgery [tw]) AND (feeding [tw] OR postoperative feeding [tw] OR enteral feeding [tw] OR nasogastric feeding [tw] OR nasojejunal feeding [tw] OR parenteral nutrition [tw] OR enteral nutrition [tw]) AND (randomized controlled trial [pt] OR controlled clinical trial [pt] OR randomized controlled trials [MESH] OR random allocation [MESH] OR double-blind method [MESH] OR single-blind method [MESH] OR clinical trial [pt] OR clinical trials [MESH] OR ("clinical trial" [tw]) OR ((singl* [tw] OR doubl* [tw] OR tripl* [tw]) AND (mask* [tw] OR blind* [tw])) OR placebos [MESH] OR placebo* [tw] OR random* [tw] OR research design [mh:noexp] OR Meta-Analysis [ptyp] OR systematic review [tw] NOT (animal [MESH] NOT human [MESH]) NOT case reports [pt] NOT Comment [PT] NOT letter [PT] NOT child [MESH] NOT infant [MESH] NOT child [MESH] NOT infant [MESH])

\section{Patient mobilisation}

(Pancreas surgery $[\mathrm{tw}]$ OR pancreas resection $[\mathrm{tw}]$ OR pancreatic surgery $[\mathrm{tw}]$ OR pancreatic resection [tw] OR distal pancreatectomy [tw] OR pancreatic left resection [tw] OR pancreaticoduodenectomy [tw] OR hepatic surgery [tw] OR hepatic resection [tw] OR liver surgery [tw] OR hepatectomy [tw] OR colorectal surgery [tw] OR colon resection [tw] OR colon surgery [tw] OR abdominal surgery [tw] OR gastrointestinal surgery [tw]) AND (mobili- sation [tw] OR mobilization [tw] OR postoperative mobilisation [tw] OR patient mobilisation [tw] OR postoperative mobilization [tw] OR patient mobilization [tw]) AND (randomized controlled trial [pt] OR controlled clinical trial [pt] OR randomized controlled trials [MESH] OR random allocation [MESH] OR double-blind method [MESH] OR single-blind method [MESH] OR clinical trial [pt] OR clinical trials [MESH] OR ("clinical trial" [tw]) OR ((singl* [tw] OR doubl* [tw] OR tripl* [tw]) AND (mask* [tw] OR blind* [tw])) OR placebos [MESH] OR placebo* $[\mathrm{tw}]$ OR random* $[\mathrm{tw}]$ OR research design [mh:noexp] OR Meta-Analysis [ptyp] OR systematic review [tw] NOT (animal [MESH] NOT human [MESH]) NOT case reports [pt] NOT Comment [PT] NOT letter [PT] NOT child [MESH] NOT infant [MESH] NOT child [MESH] NOT infant [MESH])

\section{Additional material}

\section{Additional file 1}

Survey on peri-operative standards in distal pancreatectomy. A copy of the research instrument used for the survey.

Click here for file

[http://www.biomedcentral.com/content/supplementary/17456215-10-58-S1.pdf]

\section{Additional file 2}

Meta-analyses and randomized controlled trials on perioperative management. Appendix 1 summarizes relevant data from the meta-anal$y$ ses and randomized controlled trials found by the literature search of best practice.

Click here for file

[http://www.biomedcentral.com/content/supplementary/17456215-10-58-S2.pdf]

\section{Acknowledgements}

The authors would like to thank Dylan Parker Rahe and Heather Karner for English language editing.

We highly appreciate the support of all DISPACT trial centres and we would like to thank all doctors who have participated in the survey on perioperative treatment.: Department of Surgery, Amsterdam Medical Centre, The Netherlands, Prof. D. Gouma, Dr. N. van der Gaag; Department of General, Visceral, Vascular and Thoracic Surgery, Charite Campus Mitte, Berlin, Germany, Prof. J. Müller, PD Dr. T. Junghans; Department of General, Visceral and Transplantation Surgery, Charite Campus Virchow, Berlin, Germany, Prof. P. Neuhaus, PD Dr. Matthias Glanemann; Department of General and Visceral Surgery, Sana Clinical Centre Berlin Lichtenberg, Germany, Prof. K. Gellert, Dr. F. Fritze; Department of General Surgery, St. Josef Hospital, Ruhr University, Bochum, Germany, Prof. W. Uhl, Dr. O. Belyaev; Department of General an Abdominal Surgery, Municipal Clinical Centre Dresden-Friedrichstadt, Germany, Prof. H. Witzigmann, Dr. H. Lauer; Department of General and Visceral Surgery, Albert-Ludwig University, Freiburg, Germany, Prof. U.T. Hopt, Dr. T. Keck; Department of Surgery, University Medical Centre, Gent, Belgium, Prof. X. Rogiers, Dr. F. Berrevoet; Department of General, Visceral and Transplantation Surgery, University Medical Centre, Heidelberg, Germany, Prof. W. Büchler, Dr. N. 
Rahbari; Department of General, Visceral, Trauma and Pediatric Surgery, University Medical Centre, Homburg/Saar, Germany, Prof. M. Schilling, Dr. J. Schuld; Department of General, Visceral and Trauma Surgery, Hospital of the Augustins gGmbH, Cologne, Germany, Prof. T. Beckurts, Dr. E. Pfisterer; Department of Visceral, Vascular and Transplantation Surgery, Merheim Hospital, Cologne, Germany, Prof. M. Heiss, PD Dr. C. Rudroff; Division of Surgery and Oncology, Royal Liverpool University Hospital, Liverpool, UK, Prof. J.P. Neoptolemos, Dr. M. Raraty; Department of Abdominal Surgery, University Medical Center, Ljubljana, Slovenia, Prof. S. Repse, Dr. A. Tomazic; Department of Surgery, University Clinical Centre Schleswig-Holstein, Lübeck, Germany, Prof. H.-W. Bruch, PD Dr. Dr. U. Roblick; Department of Surgery, University of Mannheim, Germany, Prof. S. Post, Dr. N. Aramin; Department of Surgery, Philipps-University Marburg, Marburg, Germany, Prof. M. Rothmund, Prof. D. Bartsch; Department of Surgery, Clinical Centre Grosshadern, University of Munich, Munich, Germany, Prof. K.W. Jauch, Dr. C. Bruns; Department of Surgery, Clinical Centre rechts der Isar, Prof. R. Siewert/Prof. H. Friess, PD Dr. Schuhmacher; Department of Surgery, University Medical School, Regensburg, Germany, Prof. H. J. Schlitt, Dr. J. Werner; Department of Surgery, Borgo Roma University of Verona, Verona, Italy, Prof. C. Bassi, Dr. G. Butturini; Department of Surgery, University of Wurzburg, Wurzburg, Germany; Prof. A. Thiede/Prof. C.-T. Germer, Dr. U. Steger; Department of Surgery, Helios Hospital Wuppertal, Germany, Prof. H. Zirngibl, Dr. S. Ganzera.

\section{References}

I. Anderson ADG, McNaught CE, MacFie J, Tring I, Barker P, Mitchell $\mathrm{CJ}$ : Randomized clinical trial of multimodal optimization and standard perioperative surgical care. Br J Surg 2003, 90:1497-I504.

2. Gervaz P, Bucher P, Scheiwiller A, Mugnier-Konrad B, Morel P: The duration of postoperative ileus after elective colectomy is correlated to surgical specialization. Int J Colorectal Dis 2006, 2I:542-546.

3. Kehlet $H$, Wilmore DW: Evidence-based surgical care and the evolution of fast-track surgery. Ann Surg 2008, 248: 189-198.

4. Wasey N, Baughan J, de Gara CJ: Prophylaxis in elective colorectal surgery: the cost of ignoring the evidence. Can J Surg 2003, 46:279-284.

5. Wichmann MW, Roth M, Jauch KW, Bruns CJ: A prospective clinical feasibility study for multimodal "fast track" rehabilitation in elective pancreatic cancer surgery. Rozhl Chir 2006, 85:169-175.

6. Allareddy V, Allareddy V, Konety BR: Specificity of procedure volume and in-hospital mortality association. Ann Surg 2007, 246: $135-139$.

7. van Heek NT, Kuhlmann KFD, Scholten RJ, de Castro SMM, Busch ORC, van Gulik TM, Obertop H, Gouma D): Hospital volume and mortality after pancreatic resection: a systematic review and an evaluation of intervention in the netherlands. Ann Surg 2005, 242:78I-8.

8. Müller MW, Friess H, Kleeff J, Dahmen R, Wagner M, Hinz U, BreischGirbig D, Ceyhan GO, Büchler MW: Is there still a role for total pancreatectomy? Ann Surg 2007, 246:966-974.

9. Birkmeyer JD, Siewers AE, Finlayson EVA, Stukel TA, Lucas FL, Batista I, Welch HG, Wennberg DE: Hospital volume and surgical mortality in the united states. N Engl J Med 2002, 346: I I 28- I I 37.

10. Bramhall SR, Allum WH, Jones AG, Allwood A, Cummins C, Neoptolemos JP: Treatment and survival in 13,560 patients with pancreatic cancer, and incidence of the disease, in the west midlands: an epidemiological study. BrJ Surg 1995, 82: I II-I I 5.

11. Neoptolemos JP, Russell RC, Bramhall S, Theis B: Low mortality following resection for pancreatic and periampullary tumours in 1026 patients: uk survey of specialist pancreatic units. uk pancreatic cancer group. Br J Surg I997, 84: I370- I 376.

12. Sohn TA, Yeo CJ, Cameron JL, Koniaris L, Kaushal S, Abrams RA, Sauter PK, Coleman J, Hruban RH, Lillemoe KD: Resected adenocarcinoma of the pancreas- 616 patients: results, outcomes, and prognostic indicators. J Gastrointest Surg 2000, 4:567-579.

13. Birkmeyer JD, Sun Y, Wong SL, Stukel TA: Hospital volume and late survival after cancer surgery. Ann Surg 2007, 245:777-783.
14. Büchler MW, Wagner M, Schmied BM, Uhl W, Friess H, Z'graggen K: Changes in morbidity after pancreatic resection: toward the end of completion pancreatectomy. Arch Surg 2003, 138: $13 \mid 0-4$.

15. Diener M, Knaebel H, Witte S, Rossion I, Kieser M, Buchler M, Seiler C: Dispact trial: a randomized controlled trial to compare two different surgical techniques of distal pancreatectomy study rationale and design. Clin Trials 2008, 5:534-545.

16. Lorenz W, Weitzel F, Sitter H: Consensus-assisted development of a study protocol on sepsis: an important difference from previous randomized trials. Theor Surg 1994, 9:63-67.

17. Knaebel HP, Diener MK, Wente MN, Büchler MW, Seiler CM: Systematic review and meta-analysis of technique for closure of the pancreatic remnant after distal pancreatectomy. Br J Surg 2005, 92:539-546.

18. Hoffmann JC, Fischer I, Höhne W, Zeitz M, Selbmann H: Methodological basis for the development of consensus recommendations. Z Gastroenterol 2004, 42:984-986.

19. Guenaga KF, Matos D, Castro AA, Atallah AN, Wille-Jørgensen P: Mechanical bowel preparation for elective colorectal surgery. Cochrane Database Syst Rev 2005:CD00 544.

20. Slim K, Vicaut E, Panis Y, Chipponi J: Meta-analysis of randomized clinical trials of colorectal surgery with or without mechanical bowel preparation. Br J Surg 2004, 91 : I I 25- I I 30.

21. Bucher P, Gervaz P, Soravia C, Mermillod B, Erne M, Morel P: Randomized clinical trial of mechanical bowel preparation versus no preparation before elective left-sided colorectal surgery. $\mathrm{Br} J$ Surg 2005, 92:409-4I4.

22. Contant CM, Hop WC, van't Sant HP, Oostvogel HJ, Smeets HJ, Stassen LP, Neijenhuis PA, Idenburg FJ, Dijkhuis CM, Heres P, van Tets WF, Gerritsen JJ, Weidema WF: Mechanical bowel preparation for elective colorectal surgery: a multicentre randomised trial. Lancet 2007, 370:21I2-2117.

23. Fa-Si-Oen P, Roumen R, Buitenweg J, Velde $C$ van de, van Geldere D, Putter H, Verwaest C, Verhoef L, de Waard J, Swank D, D'Hoore A, Croiset van Uchelen F: Mechanical bowel preparation or not? outcome of a multicenter, randomized trial in elective open colon surgery. Dis Colon Rectum 2005, 48: I509-15I6.

24. Jung B, Påhlman L, Nyström P, Nilsson E: Multicentre randomized clinical trial of mechanical bowel preparation in elective colonic resection. Br J Surg 2007, 94:689-695.

25. Pena-Soria MJ, Mayol JM, Anula-Fernandez R, Arbeo-Escolar A, Fernandez-Represa JA: Mechanical bowel preparation for elective colorectal surgery with primary intraperitoneal anastomosis by a single surgeon: interim analysis of a prospective singleblinded randomized trial. J Gastrointest Surg 2007, I I:562-567.

26. Ram E, Sherman Y, Weil R, Vishne T, Kravarusic D, Dreznik Z: Is mechanical bowel preparation mandatory for elective colon surgery? A prospective randomized study. Arch Surg 2005, 140:285-288.

27. Brown SR, Goodfellow PB: Transverse verses midline incisions for abdominal surgery. Cochrane Database Syst Rev 2005:CD005199.

28. Fassiadis N, Roidl M, Hennig M, South LM, Andrews SM: Randomized clinical trial of vertical or transverse laparotomy for abdominal aortic aneurysm repair. $\mathrm{Br} J$ Surg 2005, 92:|208-|2||.

29. Song F, Glenny AM: Antimicrobial prophylaxis in colorectal surgery: a systematic review of randomized controlled trials. BrJ Surg 1998, 85: 1232-I24I.

30. Fujita S, Saito N, Yamada T, Takii Y, Kondo K, Ohue M, Ikeda E, Moriya $Y$ : Randomized, multicenter trial of antibiotic prophylaxis in elective colorectal surgery: single dose vs 3 doses of a second-generation cephalosporin without metronidazole and oral antibiotics. Arch Surg 2007, 142:657-661.

31. Gurusamy KS, Samraj K, Davidson BR: Routine abdominal drainage for uncomplicated liver resection. Cochrane Database Syst Rev 2007:CD006232.

32. Petrowsky H, Demartines N, Rousson V, Clavien P: Evidence-based value of prophylactic drainage in gastrointestinal surgery: a systematic review and meta-analyses. Ann Surg 2004, 240: 1074-84. discussion 1084-5

33. Conlon KC, Labow D, Leung D, Smith A, Jarnagin W, Coit DG, Merchant N, Brennan MF: Prospective randomized clinical trial of the value of intraperitoneal drainage after pancreatic resection. Ann Surg 200I, 234:487-93. 
34. Sun H, Qin L, Lu L, Wang L, Ye Q, Ren N, Fan J, Tang Z: Randomized clinical trial of the effects of abdominal drainage after elective hepatectomy using the crushing clamp method. Br J Surg 2006, 93:422-426.

35. Marret E, Remy C, Bonnet F: Meta-analysis of epidural analgesia versus parenteral opioid analgesia after colorectal surgery. BrJ Surg 2007, 94:665-673.

36. Werawatganon T, Charuluxanun S: Patient controlled intravenous opioid analgesia versus continuous epidural analgesia for pain after intra-abdominal surgery. Cochrane Database Syst Rev 2005:CD004088.

37. Wu CL, Cohen SR, Richman JM, Rowlingson AJ, Courpas GE, Cheung K, Lin EE, Liu SS: Efficacy of postoperative patient-controlled and continuous infusion epidural analgesia versus intravenous patient-controlled analgesia with opioids: a meta-analysis. Anesthesiology 2005, 103:1079-1088.

38. Jørgensen $H$, Wetterslev J, Møiniche S, Dahl JB: Epidural local anaesthetics versus opioid-based analgesic regimens on postoperative gastrointestinal paralysis, ponv and pain after abdominal surgery. Cochrane Database Syst Rev 2000:CD00I893.

39. Flisberg P, Rudin A, Linnér R, Lundberg CJF: Pain relief and safety after major surgery. a prospective study of epidural and intravenous analgesia in $\mathbf{2 6 9 6}$ patients. Acta Anaesthesiol Scand 2003, 47:457-465

40. Mann C, Pouzeratte Y, Boccara G, Peccoux C, Vergne C, Brunat G, Domergue J, Millat B, Colson P: Comparison of intravenous or epidural patient-controlled analgesia in the elderly after major abdominal surgery. Anesthesiology 2000, 92:433-44I.

41. Nelson R, Edwards S, Tse B: Prophylactic nasogastric decompression after abdominal surgery. Cochrane Database Syst Rev 2007:CD004929.

42. Nelson R, Tse B, Edwards S: Systematic review of prophylactic nasogastric decompression after abdominal operations. $\mathrm{Br} J$ Surg 2005, 92:673-680.

43. Pessaux P, Regimbeau J, Dondéro F, Plasse M, Mantz J, Belghiti J: Randomized clinical trial evaluating the need for routine nasogastric decompression after elective hepatic resection. BrJ Surg 2007, 94:297-303.

44. Andersen HK, Lewis SJ, Thomas S: Early enteral nutrition within $24 \mathrm{~h}$ of colorectal surgery versus later commencement of feeding for postoperative complications. Cochrane Database Syst Rev 2006:CD004080.

45. Han-Geurts IJM, Hop WCJ, Kok NFM, Lim A, Brouwer KJ, Jeekel J: Randomized clinical trial of the impact of early enteral feeding on postoperative ileus and recovery. $\mathrm{Br}$ J Surg 2007 94:555-561.

46. Browning L, Denehy L, Scholes RL: The quantity of early upright mobilisation performed following upper abdominal surgery is low: an observational study. Aust J Physiother 2007, 53:47-52.

47. Fagevik Olsén M, Hahn I, Nordgren S, Lönroth H, Lundholm K: Randomized controlled trial of prophylactic chest physiotherapy in major abdominal surgery. BrJ Surg 1997, 84:1535-1538.

48. Mackay MR, Ellis E, Johnston C: Randomised clinical trial of physiotherapy after open abdominal surgery in high risk patients. Aust J Physiother 2005, 5 I: I II-159.

49. Connor S, Alexakis N, Garden O], Leandros E, Bramis ], Wigmore S]: Meta-analysis of the value of somatostatin and its analogues in reducing complications associated with pancreatic surgery. Br J Surg 2005, 92:1059-1067.

50. Alghamdi AA, Jawas AM, Hart RS: Use of octreotide for the prevention of pancreatic fistula after elective pancreatic surgery: a systematic review and meta-analysis. Can J Surg 2007, 50:459-466.

51. Zeng Q, Zhang Q, Han S, Yu Z, Zheng M, Zhou M, Bai J, Jin R: Efficacy of somatostatin and its analogues in prevention of postoperative complications after pancreaticoduodenectomy: a meta-analysis of randomized controlled trials. Pancreas 2008, 36:18-25.

52. Hesse UJ, DeDecker C, Houtmeyers P, Demetter P, Ceelen W, Pattyn $P$, Troisi R, deHemptinne B: Prospectively randomized trial using perioperative low-dose octreotide to prevent organrelated and general complications after pancreatic surgery and pancreatico-jejunostomy. World / Surg 2005, 29: I 325-1328.

53. Shan Y, Sy ED, Tsai M, Tang L, Li PS, Lin P: Effects of somatostatin prophylaxis after pylorus-preserving pancreaticoduodenec- tomy: increased delayed gastric emptying and reduced plasma motilin. World j Surg 2005, 29:1319-1324.

54. Hughes ES: Asepsis in large-bowel surgery. Ann R Coll Surg Engl 1972, 51:347-356.

55. Holland AJ, Castleden WM, Norman PE, Stacey MC: Incisional hernias are more common in aneurysmal arterial disease. Eur J Vasc Endovasc Surg 1996, I 2:196-200.

56. Johnson B, Sharp R, Thursby P: Incisional hernias: incidence following abdominal aortic aneurysm repair. J Cardiovasc Surg (Torino) 1995, 36:487-490.

57. Liapis CD, Dimitroulis DA, Kakisis JD, Nikolaou AN, Skandalakis P, Daskalopoulos M, Kostakis AG: Incidence of incisional hernias in patients operated on for aneurysm or occlusive disease. Am Surg 2004, 70:550-552.

58. Domínguez Fernández E, Post S: Abdominal drainages. Chirurg 2003, 74:91-98.

59. Bassi C, Dervenis C, Butturini G, Fingerhut A, Yeo C, Izbicki J, Neoptolemos J, Sarr M, Traverso W, Büchler MW: Postoperative pancreatic fistula: an international study group (ISGPF) definition. Surgery 2005, I 38:pp8-I3.

60. Kehlet $\mathrm{H}$ : Future perspectives and research initiatives in fasttrack surgery. Langenbecks Arch Surg 2006, 39 I:495-498.

6I. Schwenk W, Raue W, Haase O, Junghans T, Müller JM: "fast-track" colonic surgery-first experience with a clinical procedure for accelerating postoperative recovery. Chirurg 2004, 75:508-5I4.

62. Ramos-De la Medina A, Sarr MG: Somatostatin analogues in the prevention of pancreas-related complications after pancreatic resection. J Hepatobiliary Pancreat Surg 2006, 13:190-193.
Publish with Biomed Central and every scientist can read your work free of charge

"BioMed Central will be the most significant development for disseminating the results of biomedical research in our lifetime. "

Sir Paul Nurse, Cancer Research UK

Your research papers will be:

- available free of charge to the entire biomedical community

- peer reviewed and published immediately upon acceptance

- cited in PubMed and archived on PubMed Central

- yours - you keep the copyright 